
\title{
25 Research Square \\ Liver Cirrhosis, Etiology and Clinical Characteristics Disparities among Minority Population
}

\author{
Muhammad Abu Tailakh \\ Ben-Gurion University of the Negev
}

\section{Liat Poupko}

Ben-Gurion University of the Negev

\section{Najwan Kayyal}

Soroka Medical Center

\section{Alsana Ali}

Soroka Medical Center

Ohad Etzion

Soroka Medical Center

Heba Abu-Kaf

Soroka Medical Center

Alexander Fich

Soroka Medical Center

David Yardni

Soroka Medical Center

\section{Anat Nevo-Shor}

Soroka Medical Center

\section{Abed Abu Ganim}

Soroka Medical Center

Naim Abu-Freha ( $\square$ abufreha@yahoo.de )

Institute of Gastoenterology and Hepatology, Soroka University Medical Center, Faculty of Health

Sciencs, Ben Gurion University of the Negev https://orcid.org/0000-0002-4844-3308

\section{Research article}

Keywords: Liver cirrhosis, Arab Bedouin, southern Israel, complications, mortality

Posted Date: November 14th, 2019

DOI: https://doi.org/10.21203/rs.2.15257/v2

License: (9) This work is licensed under a Creative Commons Attribution 4.0 International License. 
Version of Record: A version of this preprint was published at Journal of Immigrant and Minority Health on August 28th, 2021. See the published version at https://doi.org/10.1007/s10903-021-01263-y. 


\section{Abstract}

Background: Liver cirrhosis (LC) is a common disease with varied primary causes and ethnic disparities. We aimed to determine the frequency, clinical characteristics, and outcomes of LC among the Arab Bedouin (AB) population in southern Israel.

Methods: This retrospective and follow-up study enrolled all patients diagnosed with LC at the gastroenterology and hepatology department or the internal medicine ward. Patient records were reviewed for demographic, clinical data, and mortality. Clinical primary outcomes were defined as death, hepatocellular carcinoma or liver transplantation.

Results: We included 820 patients 69 (8.4\%) AB and 752 (91.6\%) Jewish patients. Incidence of cirrhosis found in our study was much lower among AB patients, 5-10/100,000/year, compared with 20$40 / 100,000$ per year in Jewish patients. Age at diagnosis was $42.6 \pm 16.9$ years among Bedouins compared to $61 \pm 13$ years $(p<0.001)$ among Jews. The study included $25(36.2 \%)$ male $A B$ and $472(63 \%)$ male Jewish patients $(p<0.001)$. The most frequent causes of $L C$ among $A B$ were cryptogenic $(24.6 \%)$, hepatitis B (18.8\%) and autoimmune hepatitis (17.4\%), while hepatitis C (41.7\%), fatty liver (16.5\%) and alcoholic liver disease (15.5\%) were most common among Jewish patients. An all-cause mortality of $41.8 \%$ was found in $A B$ patients compared to $63.4 \%$ in Jewish patients $(p<0.001)$. Primary clinical outcomes were found by $34(49 \%)$ AB patients and by 510 (67.9\%) Jewish patients $(p=0.002)$, with 46 and 608 events, retrospectively.

Conclusions: The incidence of liver cirrhosis among Arab Bedouin is lower than that in the Jewish population, with disparities in etiology, age at diagnosis, presence of complications and mortality.

\section{Introduction}

Liver cirrhosis (LC) is a progressive scarring of liver tissue that occurs in advanced stages of chronic liver disease and is associated with several complications, including hepatocellular carcinoma (HCC) and mortality[1].

There are several causes of cirrhosis, some important ones being autoimmune, viral, metabolic, toxic, and cryptogenic etiologies (with no obvious cause). Alcoholic liver disease is one of the most common causes of LC worldwide. In Europe, excessive alcohol consumption accounts for $40-50 \%$ of all liver cancer cases[2].

In the Middle East, LC is still one of the top four causes of mortality [3]. Egypt, with the highest prevalence of hepatitis $\mathrm{C}$ worldwide, suffers from a high prevalence of cirrhosis complications and mortality, according to a recently published study [4-6].In addition to the aforementioned etiologies, non-alcoholic fatty liver disease (NAFLD) may also be a significant source of cirrhosis. The rise in the disease in some developing countries may be explained partially by the transition to a more Western lifestyle and diet, thus increasing the incidence of NAFLD [7]. 
In contrast with its neighbors, Israel's rates of cirrhosis are considerably lower. From 2010 to 2012, chronic liver disease was ranked as the tenth leading cause of death in males between the ages of 25 and 74 years. Only 4.2 out of 100,000 Israeli men died of chronic liver disease, with negligible rates among women [8]. However, to the best of our knowledge, little research has been conducted on liver disease in the AB population in Southern Israel.

$A B$ in southern of Israel (Bedouin) are a subgroup within the Arab minority in Israel with historical, social, and cultural uniqueness. They reside in southern Israel (the Negev desert), central Israel, and northern Israel (the Galil). The AB population in southern Israel is estimated to be 250,000 [9].

Approximately $40 \%$ of Bedouins in the Negev reside in rural areas in semi-nomadic conditions. Families live in makeshift tin shacks for extended periods of time, without basic municipal services such as supply of water and electricity. Their homes are distant from the main roads, making them difficult to access. However, the rapid urbanization of the population over recent decades has shaken the socioeconomic foundations of Arabs society. The transition from tribal nomadism to a primarily urban, Western lifestyle makes this population of special interest due to a high prevalence of diseases associated with rapid urbanization, such as diabetes mellitus, ischemic heart disease, and smoking-related illnesses [10,11].

The primary aim of this study is to conduct a retrospective review of epidemiologic and clinical data on a group of AB patients with LC and compare the data to that of Israeli Jewish patients in southern Israel.

The secondary aim is to observe the distinctions between the populations concerning LC complications, such as esophageal varices bleeding, hepatocellular carcinoma and mortality.

\section{Methods}

Patients: In this retrospective study, patients with a confirmed diagnosis of LC were selected from the databases of the department of gastroenterology and liver disease and internal medicine wards at Soroka University Medical Center (SUMC).

SUMC is a tertiary, 1100 -bed hospital located in the city of Be'er-Sheva in southern Israel. It is the sole medical center providing primary care for approximately 700,000 residents in the Southern district. Patients were diagnosed through liver biopsy results or clear clinical signs of cirrhosis (portal hypertension, ascites, esophageal varices, etc.).

\section{Data collection}

Demographic and clinical data for all participants were gathered and reviewed using a number of computerized databases from the department of internal medicine and the department of gastroenterology and liver disease in SUMC. Demographic data collected from patient medical records included age, gender, and ethnicity; clinical data included etiology of cirrhosis, laboratory values, complications, and death. The Child-Pugh score for severity of cirrhosis of each patient was calculated at 
the time of diagnosis and a second score was calculated at the time of data collection or death (in cases, patients died before collection of the data).

The Child-Pugh score calculation factored in values for albumin, international normalized ratio (INR) of prothrombin time (PT), total bilirubin, encephalopathy, and ascites.

The diagnoses of the cirrhosis etiologies were determined based on follow-up visits by a gastroenterologist/hepatologist, during hospitalization in the internal medicine department, laboratory results (hepatitis $B$ surface antigen, $\mathrm{Hb}$ surface antibody, hepatitis $\mathrm{C}$ virus antibody, viral load for hepatitis B or C, anti-mitochondrial Ab, anti-smooth muscle cell Ab, anti-nuclear Ab, ceruloplasmin, and others), imaging findings, liver biopsy, and endoscopy. The diagnosis of cirrhosis was determined in cases with significant findings support the diagnosis of cirrhosis as findings in the biopsy, portal hypertension (esophageal varices, ascites) or findings in the imaging. Cases without certain diagnosis were excluded. Finally, the data of $A B$ Bedouins and Jews were compared.

\section{Primary clinical outcomes}

Primary clinical outcomes were defined as death, hepatocellular carcinoma or liver transplantations.

The study was carried out in accordance with the principles of the Helsinki Declaration. The study protocol was approved by the Institutional Helsinki Committee.

\section{Statistical analysis}

Patient characteristics were presented as mean \pm SD for continuous variables and as percentages for categorical variables. Categorical variables were compared using the chi-square test. Continuous variables were examined with the student t-test. Continuous variables that were not normally distributed were reported as median (IQR) and compared in the Kruskal Wallis test. The rates of outcomes in followup were estimated using the Kaplan-Meier method, and differences between $A B$ and Jewish patients were assessed by the log-rank test. All statistical analyses were performed using IBM SPSS version 24 (Chicago, USA). P-values less than 0.05 were considered statistically significant.

\section{Results}

\section{Incidence of liver cirrhosis}

In general, only $8.4 \%$ of our patients' cohort were AB. In this study, we calculated the incidence of LC in the $A B$ and Jewish populations between the years 2002 and 2016. We found a high age-adjusted incidence rate in Jewish patients compared to that in AB, 20-40/100,000 per year and 5-10/100,000 per year, respectively. An unexplained drop in the curve was observed between the years 2004 and 2005 in the both 
groups, and in the last years 2010-2016, a decline in incidence in the Jewish population was observed. The age-adjusted incidence of cirrhosis among $A B$ and Jewish patients is described in figure 1.

\section{Study population}

The study included 820 participants diagnosed with LC during follow-up by a gastroenterologist/hepatologist or during hospitalization in the internal medicine ward; the mean age at diagnosis was $58.6 \pm 14.6,498(61 \%)$ patients of our cohort were male. The follow up time during the study ranged between $1-31$ years, with a mean of $8.6 \pm 4.9$ years.

The AB patients tended to be diagnosed at younger ages than Jewish patients, at a mean of $42.6 \pm 16.9$ years, as opposed to $61 \pm 13$ years in Jewish patients $(p<0.001)$.

Female $A B$ patients represented a larger portion of their group than Jewish women, $63.8 \%$ compared to $37 \%$, respectively $(p<0.001)$.

At baseline, there was no significant difference in rates of hepatitis $B$, fatty liver, Wilson disease and primary sclerosing cholangitis (PSC) as an etiology of LC between the two groups. However, more Jewish patients suffered from LC due to alcoholic liver disease (15.5\%) and hepatitis $C(41.7 \%)$, while more $A B$ patients had more cases of autoimmune hepatitis (17.4\%) and cryptogenic cirrhosis (24.6\%). Baseline characteristics of the two groups are described in table 1 . The laboratory results including the values are shown in table 2. No significant differences were found between the two groups regarding the laboratory results.

\section{Primary clinical outcome}

The primary clinical outcomes were defined as either death, hepatocellular carcinoma or transplantation. The various complications and outcomes are summarized in table 3. Importantly, more AB patients had esophageal varices and bleeding events.

No significant difference in rates of hepatocellular carcinoma or spontaneous bacterial peritonitis were found between the two groups.

Lower all-cause mortality (41.8\%) and younger age of death ( $51 \pm 15.1$ years) were demonstrated among AB patients as compared to Jewish patients $(63.4 \%$ all-cause mortality $[p<0.001]$ and $67 \pm 14.3$ years $[p<0.001])$.

One or more of the clinical primary outcomes was found in $34(49.3 \%)$ AB patients and in $510(67.9 \%)$ Jewish patients $(p=0.002)$, with a total of 46 and 608 events, respectively.

\section{Survival}


The Kaplan-Meier curve describing the survival rate among $A B$ and Jewish patients is shown in figure 2. After the fourth year of follow-up, the curve representing survival rate among $A B$ and Jewish patients diverges and demonstrates better survival rates among the $A B$ up to 15 years of follow-up (about $30 \%$ among $A B$ and $20 \%$ among Jewish patients), log rank $=0.02$.

\section{Discussion}

To the best of our knowledge, the present study is the first to investigate LC in Israel with a focus on the AB population.

LC is one of the most frequent causes of death in Europe and the Middle East; however, it ranks low on the list of causes of mortality in Israel $[3,8]$.

In the past three decades, the $A B$ of southern Israel have undergone a major shift in lifestyle. This transition from a seminomadic to Western lifestyle makes this population of special interest, relevant to research on other minorities worldwide.

One of the important findings of our research is the frequency of $L C$ among $A B, 8.4 \%$ of all patients in the cohort. This percentage is much lower than that of the $A B$ in the general population in southern Israel, which is about $33 \%$ [9]. Our data showed a significantly lower rate of incidence of cirrhosis among $A B$ compared with the incidence rate among the Jewish population during the follow-up time of the study. The low incidence may be specific to the Bedouin population of southern Israel due to their unique characteristics, while the incidence among the Jewish patients of southern Israel are largely representative of the general Israeli population.

Given the young average age of the $A B$ (about $59 \%$ of the Arabs are younger than age 19 years) [9], rates of cirrhosis might be expected to be low. However, it has also been suggested that it is the low frequency of hepatitis $C$ and alcohol liver disease among $A B$-generally the most common causes of cirrhosis-that may explain this low prevalence. Currently, since the approval of the direct-acting antivirals (DAA) for hepatitis $C$ treatment with a sustained viral clearance of over 95\% [12], the division of cirrhosis etiologies is likely to change in most countries. Hepatitis $\mathrm{C}$ may no longer be considered the main cause for cirrhosis. Rather, NAFLD, with its increasing incidence and no promising effective treatment on the horizon, as well as hepatitis B that is on the rise in some regions, are projected to climb higher on the list of common causes of cirrhosis in the near future. Both etiologies are responsible for many cases of cirrhosis in the $A B$ in southern Israel.

NAFLD has been shown to be associated with obesity in the literature, with $80 \%$ of NAFLD patients being obese $[13,14]$.The rates of obesity in the Arab population in Israel are higher than that of the Jewish population; $19.8 \%$ of Arab men and $24.3 \%$ of Arab women are obese, while the rates among Jews are $18 \%$ and $16 \%$, respectively [15]. Obesity in the AB age groups of $45-44,55-64$ and $65+$ years are $34 \%$, $47 \%$ and $45 \%$, respectively. The rates among women are higher, up to $60 \%$ in some age groups [16]. Older 
studies have shown that $29.9 \%$ of $A B$ women overall are obese and $42 \%$ are overweight based on prepregnancy body mass index $[17,18]$.

Given that obesity and hepatitis B are on the rise and that population in growing older, we project an increase in rates of $L C$ among $A B$ in the coming decades.

In contrast to the results of the present study regarding causes of cirrhosis, there are notable disparities in etiology between $A B$ and Jewish patients. We observed that the most common causes of cirrhosis among $A B$ patients were cryptogenic, hepatitis $B$, autoimmune hepatitis and NAFLD, while cases in Jewish patients were mainly derived from hepatitis C, NAFLD, alcoholic liver disease, and hepatitis B. In contrast with our results, studies in other countries mostly attributed alcoholic liver disease, NAFLD and hepatitis $C$ to the development of cirrhosis $[19,20]$.

In the Middle East, the most common factors leading to $L C$ are hepatitis $B$ and hepatitis $C$, with the highest prevalence of hepatitis $C$ in Egypt (14\%) [3-5].

When focusing on clinical characteristics, we find that a large proportion of $A B$ patients with cirrhosis were female, (64\% compared to $37 \%$ of Jewish patients), and the $A B$ patients were significantly younger at the time of diagnosis (by an average of twenty years). These findings are possibly related to the etiology, since cryptogenic and autoimmune hepatitis are more common among females [21], while hepatitis $\mathrm{C}$, alcoholic liver disease and NAFLD are more common in males.

Our data also show that more AB patients underwent liver biopsy than Jewish patients ( $42 \%$ versus $27 \%$ ), which may stem from the etiology of the cirrhosis. A large portion of $A B$ was diagnosed with cryptogenic or autoimmune hepatitis, and liver biopsies are necessary to formulate a complete and accurate diagnosis in these cases; such diagnostic methods are utilized less often in patients with hepatitis $\mathrm{C}$ or alcoholic liver disease.

Cryptogenic cirrhosis still high among $A B$ patients, part of the patients underwent liver biopsy and some of the didn't, however in these patients no cause for LC were found, but non alcoholic steatohepatitis still in the differential diagnosis, which is not diagnosed in the liver biopsy due to the advanced liver disease.

Notably, AB patients were diagnosed at a younger age, but had more esophageal varices, more bleeding esophageal varices, and more liver transplantations. There is no clear explanation for this finding; however, the younger age at diagnosis with poorer adherence to follow-up and treatment during the compensation period of the cirrhosis might be a risk factor for accelerated disease progression.

We found no differences in HCC and SBP. Approximately $13 \%$ of cirrhotic patients developed HCC in the two groups. Patients with LC have an increased risk for development of HCC and depending on the etiology and risk factors, the risk for HCC can reach $24 \%$ [22-24].

In the present study, the all-cause mortality among Jewish cirrhosis patients was significantly higher than in $A B$ patients (63.4\% compared to $41.8 \%$, respectively). In our study, $61 \%$ of all cirrhosis patients died 
throughout the follow-up time, a relatively high mortality rate.

Fleming et al. estimated a hazard ratio (HR) of 4.7 for death among patients with compensated cirrhosis and 9.7 among patients with decompensated cirrhosis compared to the general population [25].

Mortality from LC in 2010 was estimated to be approximately 1 million worldwide, a significant global health burden [26].This burden has variable geographic distribution due do the differenced in the predominant etiologies of cirrhosis in each country.

The survival rate of our cohort estimated to be higher among $A B$, about $30 \%$ in a follow-up after 15 years, compared to $20 \%$ among Jewish patients.

However, the primary clinical outcomes defined in this study were significantly higher among Jewish patients than $A B$ patients (67.9\% and $49.3 \%$, respectively), 608 versus 46 events.

Focusing on laboratory results, some laboratory values were abnormal as expected in both groups, including hemoglobin, platelets, liver enzymes, bilirubin, albumin and INR. A Child-Pugh score of 6 was assigned at diagnosis (Child $A$ ) and the last score was 7 and 8 (Child $B$ ) among $A B$ and Jewish patients, respectively.

Despite the discrepancies between the two groups regarding clinical characteristics and outcomes, no significant differences were found in the laboratory results. Importantly, $58 \%$ of AB patients had normal ALT levels and $38 \%$ had normal AST levels. However, as calculated in table 2, the standard deviation of AST was relatively high (1200), which could be related to the high AST levels (up to 7000) among several $A B$ patients recorded during previous hospitalizations. These high levels may be attributed to acute exacerbation or complication of the disease.

In summary, to the best of our knowledge this is the first study in Israel investigating cirrhosis in Bedouins in southern Israel. The strength of our study is in the large sample of cirrhosis patients, the findings of the study showed huge disparities among the $A B$ patients compared with the Jewish patients. $A B$ were diagnosed on average at a younger age, died at a younger age, and underwent more transplantations. Yet these $A B$ patients still had a lower all-cause mortality and better survival rates, possibly due to cirrhosis etiology, time of diagnosis, and the seeking of medical services.

There are some limitations of the present study: first, the retrospective design of the study, and second, the incomplete data on treatment, hospitalization and precise causes of death.

\section{Conclusion}

The incidence of liver cirrhosis is low among Arab Bedouin patients, with marked disparities in etiology, clinical characteristics, complications and all-cause mortality as compared to Jewish patients. 
Arab Bedouin patients were diagnosed at a younger age, underwent more liver transplantation, but had lower all-cause mortality rates and higher survival rates.

\section{Abbreviations}

\section{AB: Arab Bedouin}

LC: Liver cirrhosis

HCC: Hepatocellular Carcinoma

SBP: Spontanous Bacterial Peritonitis

NAFLD: Non-Alcoholic Fatty Liver Disease (NAFLD)

PBC: Primary Biliary Cholangitis

PSC: Primary Sclerosing Cholangitis

$\mathrm{Hb}$ : Hemoglobin

WBC: White Blood Cells

PLT: Platelets

ALT: Alanine transaminase

AST: Asparatate transaminase

AP: Alkaline Phosphatase

GGT: Gamma-Glutamyl Transferase

\section{Declarations}

\section{Acknowledgements}

Not applicable

\section{Ethics approval and consent to participate}

The study protocol was approved by the Institutional Ethics Committee, Helsinki committee at the Soroka University Medical Center. A written informed consent was not needed because of the retrospective design of the study. 


\section{Consent for publication}

Not applicable

\section{Availability of data and materials}

The datasets used and/or analyzed during the current study are available from the corresponding author on reasonable request

\section{Competing interests}

The authors declare that they have no competing interests

\section{Funding}

The study received no funding

\section{Author contributions:}

- Conception and design of the study: MAT, NAF, OE, AF

- Generation, collection, assembly, analysis and/or interpretation of data: NAF,MAT, NK, AL, OE

- Drafting or revision of the manuscript: NAF, HAK, DY, ANS, AAG, LP

- Approval of the final version of the manuscript: MAT, LP, NK, AL, OE, HAK, AF, DY, ANS, AAG and NAF

\section{References}

1. Byass P. The Global Burden of Liver Disease: A Challenge for Methods and for Public Health. BMC Medicine 2014;12:159

2. Jewell J, Sheron, N. Trends in European liver death rates: Implications for alcohol policy. Clin. Med. 2010;10: 259-263.

3. Ghajarieh Sepanlou S, Malekzadeh F, Delavari F et al. Burden of Gastrointestinal and Liver Diseases in Middle East and North Africa: Results of Global Burden of Diseases Study from 1990 to 2010. Middle East J Dig Dis. 2015;7(4):201-15

4. Alazawi W, Cunningham M, Dearden J, Foster GR. Systematic review: outcome of compensated cirrhosis due to chronic hepatitis C infection. Aliment Pharmacol Ther. 2010;32(3):344-355.

5. Kandeel A, Genedy M, El-Refai S et al. The prevalence of hepatitis C virus infection in Egypt 2015: implications for future policy on prevention and treatment. Liver Int. 2017;37: 45-53. 
6. Elgharably A, Gomaa A, Crossey $\mathrm{M}$ et al. Hepatitis C in Egypt-past, present, and future. International Journal of General Medicine 2017:10 1-6

7. Masarone M, Federico A, Abenavoli L, Loguercio C, Persico M. Nonalcoholic fatty liver: epidemiology and natural history. Rev Recent Clin Trials 2014;9:126-13

8. The Israeli Ministry of Health, available at:https://www.health.gov.il/PublicationsFiles/Leading_Causes_2012E.pdfaccessed at, 08, march, 2018

9. Abstract of Israel, State of Israel, Central Bureau of Statistics CBS, Jerusalem. available at www.cbs.gov.il, accessed at, 08, march, 2018.https://old.cbs.gov.il/shnaton68/st02_19x.pdf

10. Tamir O, Peleg R, Dreiher $\mathrm{J}$ et al. Cardiovascular Risk Factors in the Bedouin Population: Management and Compliance. Isr Med Assoc J 2007;9(9): 652-5.

11. Abou-Rbiah Y, Weitzman S. Diabetes among Bedouins in the Negev: the transition from a rare to a highly prevalent condition. Isr Med Assoc J 2002;4(9):687-9.

12. Asselah T, Marcellin $P$, Schinazi R. Treatment of hepatitis $C$ virus infection with direct-acting antiviral agents: $100 \%$ cure? Liver international 2018;38:1:7-13

13. Ong JP, Younossi ZM. Epidemiology and natural history of NAFLD and NASH. Clin Liver Dis 2007;11:1-16

14. Bellentani S, Saccoccio G, Masutti $F$ et al. Prevalence of and risk factors for hepatic steatosis in Northern Italy. Ann Intern Med. 2000 Jan 18;132(2):112-7.

15. Highlights of Health in Israel 2016, ICDC - Israel Center for Disease Control Medical Technology, Information and Research (MTIR) Division, Ministry of Health. Accessed at 03.03.2018, Available at https://www.health.gov.il/PublicationsFiles/Highlights_of_Health_in_Israel2016.pdf

16. Abo Rabia Y. Abstract number 1, Diabetes Mellitus among the Bedouin in the Negev. Arab Medical Association annual meeting, 30.09.2016, Beer-Sheva.

17. Fraser $\mathrm{D}$, Bilenko $\mathrm{N}$, Vardy $\mathrm{H}$ et al. Differences in food intake and disparity in obesity rates between adult Jews and Bedouins in southern Israel. Ethn Dis 2008;18:13-18.

18. Abu-Saad K, Shahar DR, Fraser D et al. Adequacy of usual dietary intake and nutritional status among pregnant women in the context of nutrition transition: the DEPOSIT Study. Br J Nutr 2012;108: 1874-1883

19. Wiegand J, Berg T. The Etiology, Diagnosis and Prevention of Liver Cirrhosis. Dtsch Arztebl Int 2013;110(6):85-91

20. Bell BP, Manos MM, Zaman A. The epidemiology of newly diagnosed chronic liver disease in gastroenterology practices in the United States: results from population-based surveillance. Am J Gastroenterol 2008;103:2727-36

21. Delgado JS, Vodonos A, Malnick $S$ et al. Autoimmune hepatitis in southern Israel: a 15-year multicenter study. J Dig Dis. 2013;14(11):611-8. 
22. Mancebo A, Gonzalez-Dieguez ML, Cadahia $V$ et al. Annual incidence of hepatocellular carcinoma among patients with alcoholic cirrhosis and identification of risk groups. Clin. Gastroenterol. Hepatol. 2013;11:95-101.

23. N'Kontchou G, Paries J, Htar M.T et al. Risk factors for hepatocellular carcinoma in patients with alcoholic or viral c cirrhosis. Clin. Gastroenterol. Hepatol. 2006;4:1062-1068.

24. Marot A, Henrion J, Knebel JF, Moreno C, Deltenre P. Alcoholic liver disease confers a worse prognosis than HCV infection and non-alcoholic fatty liver disease among patients with cirrhosis: An observational study. PLoS ONE. 2017;12:e0186715 doi: 10.1371/journal.pone.018671

25. Fleming KM, Aithal GP, Card TR, West J. All-cause mortality in people with cirrhosis compared with the general population: a population-based cohort study. Liver Int. 2012;32(1):79-84.

26. Mokdad A, Lopez A, Shahraz S et al. Liver cirrhosis mortality in 187 countries between 1980 and 2010: a systematic analysis. BMC Medicine 2014,12:145

\section{Tables Legend}

Table 1: Basic Demographic and Clinical Characteristics of the included patients

Table 2: Summary of the laboratory results among Arab Bedouin and Jewish cirrhosis patients

Table 3: Clinical complications and Outcomes comparison between two study groups

\section{Tables}

Table 1: Basic Demographic and Clinical Characteristics of the included patients 


\begin{tabular}{|c|c|c|c|}
\hline \multicolumn{3}{|l|}{$P$ value } & \multirow[t]{3}{*}{ Characteristics } \\
\hline & Jews & Arabs & \\
\hline & $\mathrm{N}=751$ & $\mathrm{~N}=69$ & \\
\hline$<0.001$ & $(63.0) 472$ & (36.2) 25 & Gender - Male \\
\hline$<0.001$ & $66.4 \pm 12.7$ & $49.9 \pm 14.8$ & Age \\
\hline$<0.001$ & $61 \pm 13$ & $42.6 \pm 16.9$ & Age at diagnosis \\
\hline \multirow[t]{2}{*}{0.008} & $(27.1) 201$ & $(42) 29$ & Liver biopsy \\
\hline & & & Etiology of cirrhosis \% \\
\hline 0.16 & (12.8) 96 & (18.8) 13 & Hepatitis B \\
\hline$<0.001$ & $(41.7) 313$ & (14.5) 10 & Hepatitis C \\
\hline \multirow[t]{2}{*}{0.89} & $(16.5) 124$ & $(15.9) 11$ & Non-Alcoholic Fatty Liver Disease (NAFLD) \\
\hline & & & Wilson Disease \\
\hline 0.46 & $(0.7) 5$ & $(1.4) 1$ & Alcoholic Liver Disease \\
\hline$<0.001$ & $(15.5) 116$ & $(1.4) 1$ & Autoimmune Hepatitis \\
\hline$<0.001$ & (1.3) 10 & $(17.4) 12$ & Primary Biliary Cholangitis (PBC) \\
\hline \multirow[t]{2}{*}{0.007} & $(0.8) 6$ & $(4.3) 3$ & Primary Sclerosing Cholangitis (PSC) \\
\hline & & & Cryptogenic \\
\hline 0.67 & $(0.3) 2$ & $0(0)$ & Others \\
\hline \multirow[t]{2}{*}{$<0.001$} & $(9.1) 68$ & $17(24.6)$ & \\
\hline & $9(1.1)$ & $(1.4) 1$ & \\
\hline
\end{tabular}

Table 2: Summary of the laboratory results among Bedouin Arab and Jewish cirrhosis patients (mean $\pm \mathrm{SD}$, unless otherwise stated) 


\begin{tabular}{llll}
\hline P value & Jews & Arabs & Characteristics \\
& $\mathrm{N}=749$ & $\mathbf{N}=69$ & \\
\hline 0.44 & $11.06 \pm 2.4$ & $10.8 \pm 2.8$ & Hemoglobin $(\mathrm{Hb})(\mathrm{gr} / \mathrm{dl})$ \\
\hline 0.23 & $9.1 \pm 7.9$ & $8 \pm 7.4$ & White Blood count (WBC) $\left(10^{\square} 3 / \mathrm{ul}\right)$ \\
\hline 0.09 & $119 \pm 78.2$ & $102 \pm 70.4$ & Platelets $\left(10^{\square} 3 / \mathrm{ul}\right)$ \\
\hline 0.25 & $74 \pm 240$ & $168 \pm 662$ & Alanine transaminase (ALT) (U/L) \\
\hline 0.29 & $155 \pm 623$ & $310 \pm 1205$ & Asparatate transaminase (AST) (U/L) \\
\hline 0.29 & $166 \pm 159$ & $187 \pm 176$ & Alkaline Phosphatase (AP) (U/L) \\
\hline 0.05 & $124 \pm 148$ & $97 \pm 103$ & Gamma-Glutamyl Transferase (GGT) (U/L) \\
\hline 0.95 & $4 \pm 6.8$ & $4 \pm 5.8$ & Bilirubin- Total - last (mg/dL) \\
\hline 0.71 & $2.02 \pm 3$ & $2.1 \pm 2.5$ & Bilirubin-Total - at Diagnosis \\
\hline 0.25 & $2.9 \pm 0.8$ & $3.1 \pm 0.9$ & Albumin - last (gr/dL) \\
\hline 0.01 & $3.4 \pm 0.6$ & $3.2 \pm 0.7$ & Albumin - at Diagnosis (gr/dL) \\
\hline 0.20 & $1.6 \pm 0.8$ & $1.8 \pm 1.1$ & INR-Last \\
\hline 0.03 & $1.3 \pm 0.38$ & $1.4 \pm 0.39$ & INR- at Diagnosis \\
\hline 0.61 & $137 \pm 8$ & $138 \pm 5$ & Sodium (mEq/L) \\
\hline 0.16 & $6(5 ; 8)$ & $6(5 ; 8)$ & Child-Pugh score- at Diagnosis (IQR) \\
\hline 0.39 & $8(5 ; 10)$ & $7(5 ; 10)$ & Child-Pugh score last (IQR) \\
\hline & & &
\end{tabular}

Table 3: Clinical complications and Outcomes comparison between two study groups 


\begin{tabular}{llll}
\hline P value & \multicolumn{3}{c}{ Characteristics } \\
& Jews & Arabs & \\
& $\mathbf{N}=749$ & $\mathbf{N}=69$ & \\
\hline 0.003 & $(57.2) 416$ & $52(75.4)$ & Esophageal varices \\
\hline 0.02 & $(29.6) 206$ & $(42) 29$ & Esophageal varices bleeding \\
\hline 0.86 & $(13.8) 103$ & $(13) 9$ & Hepatocellular Carcinoma (HCC) \\
\hline 0.06 & $48 \pm 28$ & $56 \pm 9$ & HCC - Age at diagnosis \\
\hline 0.91 & $(23.8) 176$ & $(23.2) 16$ & Spontaneous Bacterial Peritonitis (SBP) \\
\hline 0.75 & $131(17.5)$ & $11(15.9)$ & Hepatorenal Syndrome \\
\hline 0.84 & $79(11.9)$ & $6(9.5)$ & Hepatorenal Syndrome type 1 \\
\hline 0.54 & $4(0.5)$ & $0(0)$ & Hepatopulmonary \\
\hline 0.03 & $(4.3) 32$ & $(10.1) 9$ & Transplantation \\
\hline$<0.001$ & $(63.4) 473$ & $(41.8) 28$ & Death \\
\hline$<0.001$ & $67 \pm 14.3$ & $51 \pm 15.1$ & Age of death \\
\hline 0.002 & $510(67.9)$ & $34(49.3)$ & Primary Outcome \\
\hline & & & (Death, HCC or transplantation) \\
\hline
\end{tabular}

\section{Figures}




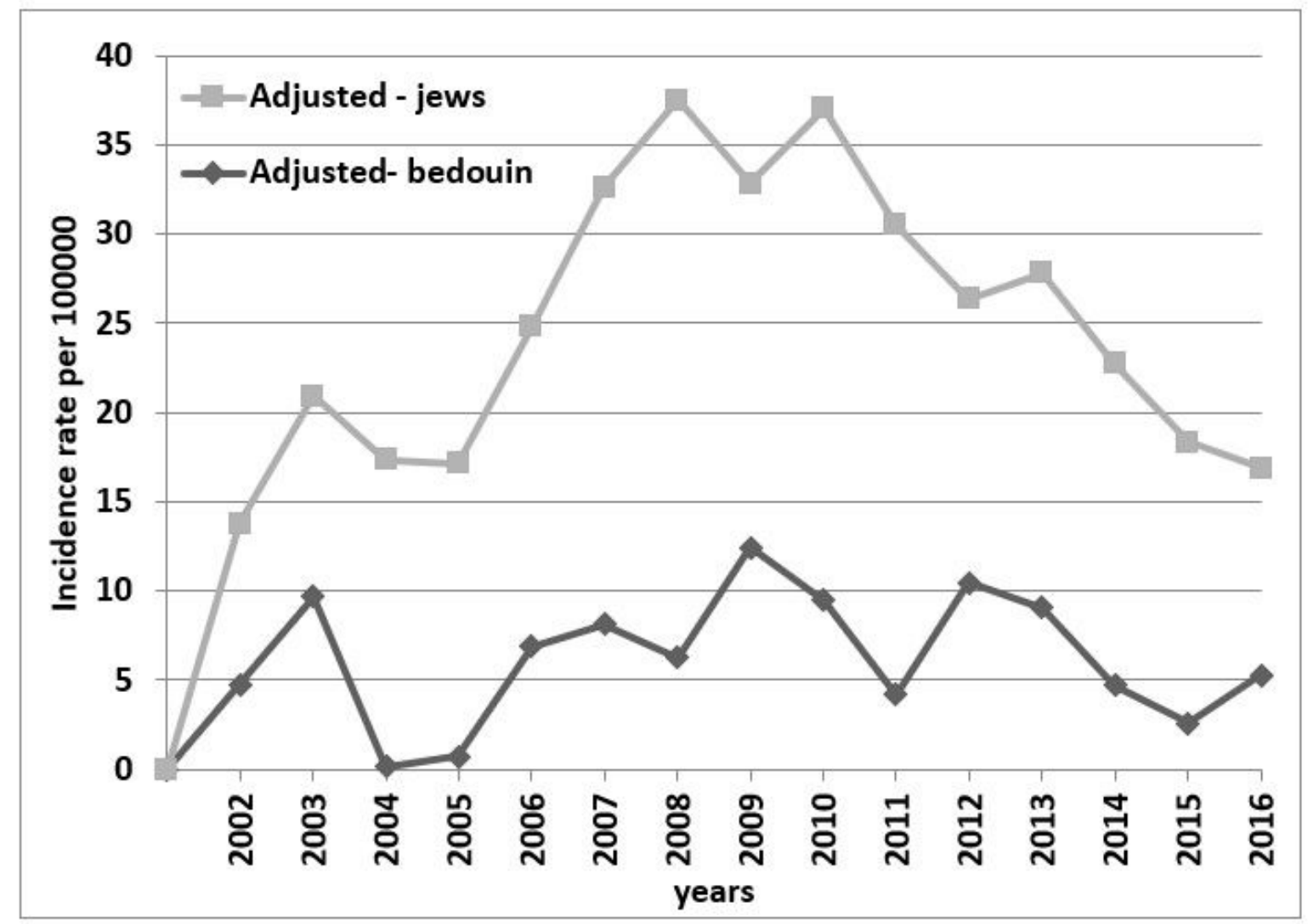

Figure 1

Age-adjusted incidence of cirrhosis among Arabs (Bedouin) and Jews population 


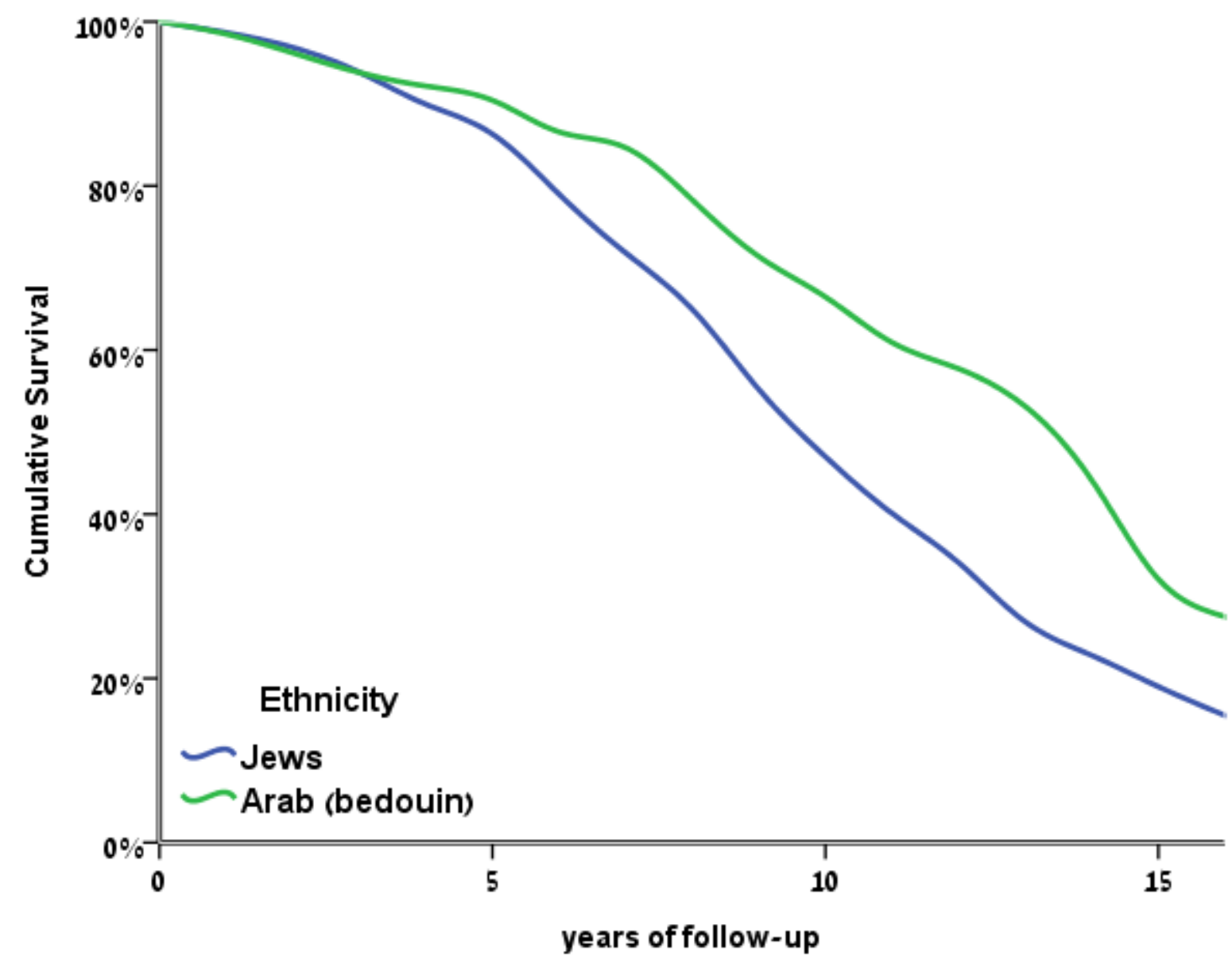

Figure 2

Kaplan-Meier survival analysis among Arab Bedouin and Jewish patients

\section{Supplementary Files}

This is a list of supplementary files associated with this preprint. Click to download.

- Table2.docx

- Table1.docx

- Table3.docx 